\title{
Paradigmatic framework of the development of modern economic theory: peculiarities of revision
}

\author{
Vitaly Biryukov, Nikolai Alekseev*, Natalia Gerasimova, and Svetlana Ilchenko \\ Omsk Humanitarian Academy, $4^{\text {th }}$ Chelyuskintsev str., 2a, 64405 Omsk, Russia
}

\begin{abstract}
The article considers the problems of revising the paradigmatic framework of the development of modern economic theories. The study is based on the use of constructivist methodology, which allows studying meaningfully the processes of economic development as a complex system. The emergence of the modern paradigmatic framework of economic theories is interpreted as a result of the use of an eclectic combination of elements of individualistic and holistic methodologies. The paper considers the issues of transition to the research paradigm of studying the links between economic relations, values and institutions, taking into account the diversity of economic motives and interests of subjects, which have the ability to create collective forms of economic activity.
\end{abstract}

\section{Introduction}

The radical changes taking place in the modern economy give rise to new challenges, the answers to which require the development of more realistic economic theories [1]. At the same time, it is important to take into account that the search for research strategies today is determined by the peculiarities of the modern stage in the evolution of economic knowledge, which emerged at the end of the last century as a result of the methodological turn. This turn is associated with the study of economics as a complex system and contributes to the fact that the dominant neoclassical system is replaced by a new, more pluralistic mainstream [2, $\mathrm{p}$. 70]. In this regard, the opposition of the mainstream, represented by neoclassical theory, to heterodox theories loses its former significance.

Nowadays, the economic science moves to the study of complex systems possessing emergent properties, the key role in their understanding is played by history and institutions [3, pp. 87-88]. The modern stage in the development of research strategies is characterized by the formation of their new quality, which is determined by the features of competition between the neoclassical direction that emerged in the last century, on the one hand, and the cultural-historical and institutional direction, on the other hand. Representatives of these directions proposed different ways of solving the key problem of studying economics, relying on alternative individualistic and holistic methodologies. In order to clarify the formation logic of the paradigmatic framework of the current vision of the problem field, it is important

\footnotetext{
*Corresponding author: oeip@mail.ru
} 
to consider the peculiarity of the methodological consensus that emerged under the influence of the normative turn and the turn in the study of economics from the position of complexity.

Since the 1940s, economic theory has been dominated by the notion that rational choice theory, which is at the core of the economic mainstream, is an attempt to develop a positive theory. At the same time, largely due to numerous empirical studies, reasoned justifications for the limited rationality of subjects' economic behavior have emerged. Because of the normative turn, today many supporters of the rational choice theory and its critics consider this theory as a normative rather than a positive theory of economic research [4]. However, competing approaches have failed to create a clear alternative to this theory. In this regard, a paradoxical situation arose: not only supporters of the economic mainstream, but also many representatives of heterodox theories developed an explicit or implicit understanding of subjects' economic motives based on individualistic methodology. Recognition of the rational choice theory as normative one contributes to the creation of more flexible research strategies, taking into account the peculiarities of studying the economy as a complex system. These features are determined primarily by the fact that the micro-foundations of complex systems depend on the context, and they should be studied in the context of the established system [3, p. 88]. The understanding of the individualistic methodology limitation and the desire to describe the subjects' economic behavior in a complex system of relations determines the use of elements of the holistic methodology in order to consider the influence of socio-cultural phenomena on their behavior.

It is impossible to explain the endogenous nature of collective forms of economic activity (common goals, values, institutions, etc.) within the framework of individualistic methodology because of the impossibility of making collective decisions (C. Arrow's impossibility theorem). Therefore, modern research strategies are predominantly created based on a dualistic model of describing economic reality, which allows us interpreting the exogenous influence of certain socio-cultural phenomena on the subjects' individualistic behavior, relying on an eclectic combination of elements of individualistic and holistic methodologies. This determines the formation of a common paradigmatic framework of theoretical and empirical research, cognitive capabilities and diagnostic limits.

According to the dualistic model, the subjects' behavior in the economy is formed under the influence of economic and non-economic motives. The economic motive is considered as individualistic, and the non-economic motive is considered as collective, connected with the fact that the economy is within the socio-cultural environment and experiences its exogenous corrective influence. Changes in the economic mainstream allow the adoption of many methods related to complexity theory. Consequently, notions on subjects are being adjusted to account for limited rationality, heterogeneity, learning capacities, etc. At the same time, the neoclassical model is also used to solve many specific problems; in doing so, neoclassic system competes with modern mainstream theories in the formal model sophistication. Different versions of institutionalism have now emerged with different theories of institutional change. Today, many supporters of neo-institutionalism and the original (old) institutionalism adhere to individualistic and holistic positions; as a result, various intersections have formed, and the boundaries have become blurred [5].

Neo-institutional theory, which has established in the last decade as the leading economic mainstream, with all its advantages and disadvantages, proceeds from a vision of the problem field lacking a value component. In neo-institutional theory, individual action and transactional processes are described based on the established norms and rules, so neoinstitutionalism tends toward a structuralism logic. In recent years, the search for more realistic interpretations of the role of institutions in economic development has been accompanied by an increase in critical studies that point to the numerous systemic flaws of neo-institutionalism [6]. However, the developed conceptual approaches associated with the 
transition to the post-institutional paradigm usually do not go beyond the dominant vision of economic reality [7].

The ongoing changes in the modern economic science are accompanied by an intensification of researches within the value-oriented direction. At the same time, the attention to the study of the mechanism of culture influence on the economy increases, since the variables reflecting its features determine many economic decisions and the development of economic processes [8]. Within the framework of this direction, various concepts are being developed, the origins of which in many respects go back to the Aristotelian tradition of considering a human as a social being; to the G. Schmoller's ideas on the role of cultural and ethical factors in the formation of economic activity and institutions, as well as to T. Veblen's theory on the mechanisms of evolution of institutions. In the conducted researches, attention is paid to the important role of ideas, values, ideology, ethical norms and traditions in shaping models of people's economic activity and economic development. On this basis, the concepts of the connection between culture and economy, moral economy, socio-economics, etc. are proposed, but even within these concepts, cultural variables act as exogenous factors, adjusting egoistic behavior.

\section{Methodology and research methods.}

The emerging methodological turn in the economic mainstream has enriched economic science. It allows developing various concepts related to the desire to take into account the influence of systemic relations on economic behavior with the help of various methods of the complexity theory. At the same time, the current research tradition is based on attempts to "work around" the dichotomy of individualism and holism by means of a dualistic model. Meanwhile, the question remains whether people can act in accordance with the economic motives of the "maximizing person" according to the rational choice theory.

In order to reconsider the logic of forming the paradigmatic framework of the development of modern economic theories, it is proposed to use the system approach, which allows studying multidimensional and nonlinear processes of economic development as a special class of complex system with emergent properties, as well as interdisciplinary approach, which is based on the constructivist methodology of studying socio-cultural reality, taking into account its intersubjective nature. The constructivist approach, emerged at the end of the last century under the influence of the turn to culture, is now one of the leading research directions in the humanities and social sciences, contributing to radical revision of the research tools and conceptual construct, as well as to the emergence of new fields of knowledge. It is expedient to change the paradigmatic framework of vision of economic reality in accordance with the intersubjective nature of economy and based on consideration of its subjects as carriers of economic culture, which allows abandoning the purposiverational model and move to the value-rational model of economic thinking and behavior of subjects, eliminating the excesses of individualistic and holistic methodologies. In this regard, there is an opportunity to refocus the research view of what is happening in the economy based on the diversity of economic motives and interests of subjects, having the ability to create collective forms of economic activity, taking into account the challenges of increasingly complex economic reality.

\section{Results and Discussion}

The study of economic reality as intersubjective in nature focuses on the consideration of subjects as structurally, culturally and institutionally rooted therein, within the established context of their economic interactions. Subjects' economic behavior is formed as a result of 
comprehension of business environment based on economic culture and economic values forming its core. Thus, economic culture, acting as a component of society's culture, exists and changes, since subjects are its carriers and creators. Relying on positivism and methodological individualism determines the use of research strategies, in which the problem of diversity of economic values is methodologically excluded; the economic problematic is reduced to the problem of coordination of already existing goals. Therefore, the diversity of ways, in which the value component enters into economic evaluation, is not taken into account.

The change in the paradigmatic framework of the vision of the economy implies the consideration of people's economic activity based on its inclusion in various economic relations evolving in time and space, which are accompanied by the formation of such intangible structures as economic institutions. The established traditions of exogenous interpretation of institutions lead to various dilemmas of the theory of institutions and institutional changes; the multiplicity and uncertainty of structuring the institutions contributes to the emergence of numerous versions of the concept of institution. Thus, within the institutional direction of research, the label "institution" is pinned to everything; while in most empirical works of the socio-cultural direction, institutions are understood as formal institutions, and informal institutions (or informal rules) appear in the form of values and beliefs (or culture) [8, p. 902].

The problem of constructing economic institutions cannot be satisfactorily solved based on exogenous interpretation of their nature. The subjects of the economy and its structures are a dual whole, the structural properties of economic systems act as a prerequisite and result of their economic and communicative practices. The systemic connectivity of the processes taking place in the economy is supported by the combination of common and individual features in the behavior of various actors, which determines the dual nature of their economic values, motives, interests, goals and results of activity. The dualism of subjects' economic culture allows creating relatively stable economic relations in the form of informal and formal institutions of economy, supported by incentive and coercive motivations based on coordination of value-normative ideas. At the same time, the mechanism of reproduction and transformation of institutions includes the processes of internalization and legitimization, associated with the evaluation by subjects of the level of trust in institutions as fair, mutually beneficial and effective.

Reconsidering the concept of institution involves abandoning the dominant notion that minimization of transaction costs is the main function of economic institutions and the central problem of neo-institutional theory [9]. Institutional choice affects the structure of economic motives of transformational and transactional processes and the generation of cumulativesynergetic effects, depending on the ratio of emerging aggregate benefits and expenses. The approach under consideration presupposes the study of economy as a complex system of economic relations and connections, the transformation of which takes place in the process of interaction of subjects possessing many economic motives and a corresponding set of criteria of economic choice of ways of coordination. Market exchange is only one of many ways of rational actions.

The proposed approach focuses on the consideration of systemic changes occurring within the national economy as emerging based on diverse and frequently conflicting interactions between actors who differ in their economic and value perception of reality, as well as in their power, financial and other types of resources. However, successful economic change is impossible without the creation of a common conventional framework. In this regard, economic practices at the surface level of reality are formed under the influence of processes occurring at the deep and intermediate levels, that is, value-normative and institutional-instrumental ones [10]. 
At the value-normative level, the search for economic ideas and justification of preferable directions of economic policy based on the peculiarities of actors' comprehension of reality takes place. Within the framework of neo-institutionalism, the peculiarity of people's economic thinking and behavior is explained by the influence of ideas and ideologies determined by institutions [11, p. 143]. However, ideas and ideologies can play not only an instrumental role, but also be the key factors in the choice of institutional policy. The formation of generally recognized priorities and methods of economic change requires justification of their overall benefit and fairness. In this regard, the expediency of supposed economic ideas and programs is inevitably supported by their conformity with the principles of common good, justice and efficiency, which leads to a discussion of ideologies expressing the divergence in the economic worldview of people's vision of a generalized image of the economy, in which cognitive, normative and symbolic components are added into. At the same time, under the influence of the ideas and ideologies of the interacting groups and the resulting balance, a value-normative compromise is created, usually in the form of somewhat distorted national "lenses". Economic ideologies, ideas, values, mythologemes, and other economic and cultural phenomena become endogenous factors in people's economic activity if they are aware of them and believe in their significance.

At the institutional-instrumental level, the state constructs the most important economic institutions within the framework of the achieved value-normative compromise; this often results in certain advantages of access to resources for the dominant groups, which determine the specifics of the transformation of different spheres and sectors of the economy. The systemic connectivity of value and institutional changes is not rigid, a variety of structural contradictions and imbalances of interests can arise in the real economy. The dependence of institutions on values gives rise to the formation of a legitimate hallway for institutional policy, since the erosion of institutions and deinstitutionalization appear at a low level of trust. Economic policy must help to maintain at least a minimum level of justice; otherwise, there would be chaos.

The ongoing radical changes, associated with the increasing role of digital technologies, contribute to the creation, through platforms and systems, of a qualitatively new channel for seeing the world and disseminating values, which is becoming one of the most important technologies of the Fourth Industrial Revolution. Various changes in the institutional environment carried out by individual and collective subjects result in creating more flexible forms of organizational and economic networks of different scales and types, coordinating the activities of private companies, non-profit organizations, and government agencies. At the same time, the development of new business formations is influenced by the valuenormative compromise that defines the framework and logic of change in institutional regulators.

The success of economic policy depends on the interest balance level in its implementation, supported by incentive and coercive methods. Significant imbalance of interests generates a cause-effect chain associated with the reduction of incentives for economic development, strengthening the role of rent-oriented behavior and coercive methods. The formation of structural dysfunctions, the trajectory of sluggish economic growth, the development of stagnation and crisis phenomena in the economy often accompany these events.

Today much attention is paid to the importance of developing theories that take into account different types of influence of the preceding trajectory and use a wide class of models and analytical tools [2, p. 70]. Refusal to interpret the evolution of the economy based on reproduction of the established type of connection between old and new institutions (path dependency) requires the recognition of the possibility of different trajectories of endogenous changes of values, institutions and various types of determination (path determinacy), within which a qualitatively new cause-effect chain of reproduction of economic and cultural codes 
is formed. The peculiarities of the national economy evolution are largely determined by the ability of state management to create a cognitive-value framework of economic thinking with regard to the changing realities, to change on this basis the priorities and methods of economic policy, the structure of motives of economic activity and interests of different groups of subjects. In this regard, economic changes can take place under the influence of endogenous reasons either within the framework of the earlier established value-normative matrix, which determines the development trajectory, or as a result of its change. This approach allows explaining the fact that reforms can be beneficial for elites, which previously blocked them [10, 12].

The project of globalization and liberalization based on a simplified understanding of economic processes, which has emerged over the past three decades, has strengthened the role of key players in financial markets and an increase in contradictions due to the inability to solve problems in a changing world situation. Critics of this project point out that even before the pandemic its crisis was clearly revealed, because the fundamental causes of the preceding global financial and economic crisis were not addressed [13]. In this regard, globalization has not become inclusive. The pandemic with its far-reaching consequences, overlapping with the global processes of technical and economic paradigm change, acted as a detonator of the crisis. In many countries affected by the pandemic, temporary restrictions on civil liberties may persist even after it has been overcome [14]. This raises the threats of the increasing role of information, digital and epidemiological technologies in strengthening human control. Developing a response to the new threats requires the implementation of alternative ideas and projects contributing to the implementation of adequate changes in the dominant worldview and value-normative foundations of society, the construction of an inclusive model that ensures a more equitable and sustainable economic development based on a balance of interests.

\section{Conclusion}

1. Going beyond the paradigmatic framework of the development of mainstream economic theories requires reconsidering traditional approaches and forming a new "categorical grid" in accordance with the constructivist logic based on a change in research methods.

2. The use of the value-rational model of economic behavior creates cognitive prerequisites for the search and discussion of research strategies to study the factors and mechanisms of economic change based on the consideration of values and institutions as endogenous regulators of the economy, as well as the diversity of their system-dynamic relationships.

3. When analyzing the processes of transformation of the national economy, it is important to consider that economic practices at the surface level of reality are formed under the influence of changes occurring at the deep and intermediate levels, that is, valuenormative and institutional-instrumental ones.

4. In the course of economy evolution, a variety of trajectories can emerge as a result of the different types of connections between old and new institutions, which suggests using path determinacy approach instead of the conceptual approach of path dependency.

5. The success of national economic development depends on the choice of the valueinstitutional model and the resulting level of balance between general and private economic interests, supported by incentive and coercive methods. 


\section{References}

1. P. Krugman, Arguing with zombies: Economics, politics, and the fight for a better future, 412 (2020)

2. D. W. Hands, Erasmus Journal for Philosophy and Economics, 8(1), 61 (2015)

3. D. Colander, Economic issues, 1, 84 (2009)

4. W. Hands, Economic issues, 10, 52 (2012)

5. G. M. Hodgson, Journal of Institutional Economics, 10(4), 591 (2014)

6. D. P. Frolov, Economic issues, 5, 107 (2020)

7. V. V. Biriukov, The Science of Person: Humanitarian Researchers, 14(3), 158 (2020)

8. A. Alesina, P. Giuliano, Journal of Economic Literature, 53(4), 898 (2015)

9. J. B. Jr. Rosser, M. V. Rosser, Evolutionary and Institutional Economic Review, 14(2), 415 (2017)

10. V. Biriukov, Society and Economy, 4, 5 (2020)

11. D. North, Institutions, Institutional Change, and the Functioning of the Economy, 190 (1997)

12. S. Mukand, D. Rodrik, NBER Working Paper, 24467, 46 (2018)

13. J. E. Stiglitz, People, power, and profits: Progressive capitalism for an age of discontent, 366 (2019)

14. Gzh. V. Kolodko, Economic issues, 5, 25 (2020) 